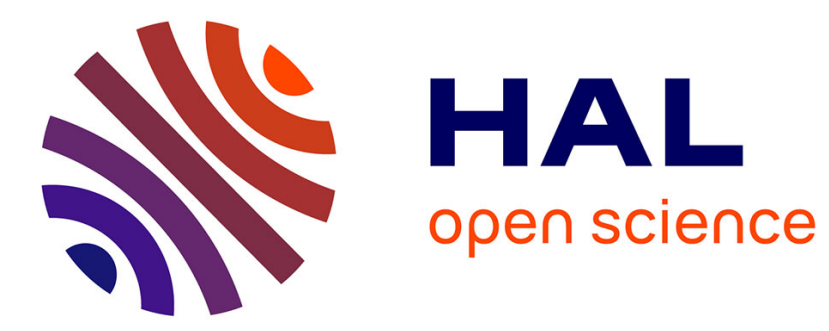

\title{
Effets de la migration sur les femmes et sur les rapports sociaux de sexe: au-delà des visions binaires
}

Nasima Moujoud

\section{To cite this version:}

Nasima Moujoud. Effets de la migration sur les femmes et sur les rapports sociaux de sexe: au-delà des visions binaires. Cahiers du CEDREF, 2008, pp.57-79. 10.4000/cedref.577 . halshs-00375578

\section{HAL Id: halshs-00375578 \\ https://shs.hal.science/halshs-00375578}

Submitted on 15 Apr 2009

HAL is a multi-disciplinary open access archive for the deposit and dissemination of scientific research documents, whether they are published or not. The documents may come from teaching and research institutions in France or abroad, or from public or private research centers.
L'archive ouverte pluridisciplinaire HAL, est destinée au dépôt et à la diffusion de documents scientifiques de niveau recherche, publiés ou non, émanant des établissements d'enseignement et de recherche français ou étrangers, des laboratoires publics ou privés. 


\title{
Effets de la migration sur les femmes et sur les rapports sociaux de sexe : au-delà des visions binaires
}

\author{
NASIMA MOUJOUD
}

Après la question des déterminants du départ des femmes, la littérature sociologique sur femmes ou genre et migration s'intéresse à la question des effets de la migration sur les migrantes (Oso-Casas, 2007). Il s'agit principalement de savoir si la migration représente ou non un "progrès » pour elles. Par exemple, l'un des enjeux majeurs des travaux féministes anglophones est d'examiner si la migration contribue à une " émancipation " des migrantes ou au contraire à les retenir dans une position de dominées (Claudine Attias-Donfut ; Catherine Delacroix, 2004). Cependant, plusieurs problèmes se posent. D'abord, le manque de travaux spécialisés et la dispersion des éléments autour de cette question, tout particulièrement en français et/ou sur la France. Ensuite, le cadre trop étroit dans lequel ces questions sont posées. En particulier, on verra ici comment un modèle binaire empêche la compréhension des phénomènes analysés. En effet, beaucoup de travaux opposent de manière simpliste société d'origine et société d'arrivée, empêchant l'émergence d'une véritable analyse globale des dynamiques de changement et d'oppression/exploitation que révèle le processus migratoire tant dans les sociétés d'arrivée que de départ. De plus, la focalisation sur certaines catégories de migrantes (notamment venues dans le cadre du regroupement familial) occulte de nombreuses autres réalités, comme nous le verrons en évoquant la situation de femmes venues seules et privées de papiers et de droits. Ensuite, la plupart des analyses se limitent aux conditions au sein de la famille et des " communautés » migrantes, négligeant de prendre en compte l'ensemble des rapports sociaux de sexe, ainsi que les autres rapports sociaux de pouvoir, tant dans la société d'origine que dans celle d'arrivée. Enfin, le rôle des États de départ et d'arrivée, tout comme les similarités dans les changements que connaissent les femmes dans les diverses sociétés, ne sont pas intégrés dans la réflexion.

Laissant de côté les travaux spécialisés produits dans les pays d'émigration, je me centrerai ici sur des textes publiés dans des pays occidentaux d'immigration, en particulier des textes francophones qui présentent des analyses à partir du cas français. Je ne ferai pas un examen exhaustif de la complexité de la question des effets de la migration sur les femmes. J'insisterai sur le fait que les éventuels effets positifs de la migration pour les migrantes se décident dans le champ des rapports sociaux de pouvoir : ce sont eux qui doivent être placés au coeur de l'analyse. Je démontrerai la nécessité de prendre résolument nos distances par rapport à une vision binaire simpliste et travailler sur les stratégies des migrantes et des personnes ou groupes qui leur sont proches et/ou solidaires.

Pour ce faire, je procèderai en trois temps :

1) Je présenterai un état des lieux sur la question des effets de la migration sur les migrantes, à partir d'un corpus de textes diversifiés qui couvrent la période des années 1990 et 2000. Nous verrons que les approches sont variées, se distinguent et parfois se contredisent. La présentation de cette production nous permettra de mettre en évidence le poids de la vision binaire entre les sociétés de départ et d'arrivée.

2) Je soulignerai les implications de la vision binaire sur l'analyse des effets de la migration sur les femmes, qui incluent l'altérisation des femmes, l'occultation de la 
domination et la non-prise en compte des dynamiques de changement dans les sociétés de départ.

3) À partir d'un point de vue que nous ne sommes pas habitués à entendre, celui des migrantes en situation irrégulière parties seules, je voudrais reformuler la question des effets de la migration sur les femmes, en l'analysant sous l'angle des initiatives que les migrantes sont amenées à prendre pour contourner les effets de la domination ${ }^{1}$.

\section{Comment se présente la question des effets de la migration sur les migrantes?}

La question des effets de la migration sur les femmes se présente fréquemment sous l'angle des changements que connaissent les migrantes (et non pas toutes les femmes) ou de leurs acquis dans la société d'immigration, par rapport à leur société d'origine. Souvent fondée sur la distinction entre deux types de sociétés, cette vision domine dans les travaux sur les migrantes. Elle est binaire et se caractérise parfois par un paradigme évolutionniste - qui en constitue la manifestation la plus criante. Ce paradigme évolutionniste, empreint de stéréotypes sur les (é)migrantes et les sociétés d'origine, a été largement critiqué par les spécialistes de la migration, comme Anne Golub, Mirjana Morokvasič et Catherine Quiminal (1997), ainsi que Laura Oso et Christine Catarino (1997). Ces deux dernières affirment que ce paradigme laisse croire que l'insertion en immigration suffirait à l'émancipation des migrantes venues du «Sud» (Oso et Catarino, 1997). Contrairement au paradigme évolutionniste, la vision binaire dans son ensemble demeure importante et moins questionnée. Elle n'est d'ailleurs pas propre aux travaux sur l'immigration ni à ceux publiés à partir de l'Occident. Elle est portée par divers analystes indépendamment de leur « race » ou de leur sexe. Par exemple, c'est à travers le paradigme de la binarité modernité/tradition que se développent des stéréotypes sur les rurales et les migrantes de classes défavorisées au Maroc (Mernissi, 1981) ou dans le « discours arabe sur l'émancipation » (Hoda al Sadda, 2004). La vision binaire occupe aussi une place importante dans d'autres champs de recherche, mais ce texte se concentre sur ses manifestations dans les travaux sur femmes et migration.

\section{A. L’approche évolutionniste}

L'analyse des changements dans la vie des migrantes s'est longtemps appuyée sur le paradigme de passage de la tradition à la modernité, comme l'indique Stéphanie Condon (2000). L'idée d'un changement linéaire de la situation des migrantes s'exprime ainsi de diverses manières dans des textes qui établissent d'emblée une opposition entre les sociétés (ou parfois les «cultures ») de départ et d'arrivée. En France, elle se retrouve tant à propos des migrantes que de leurs descendantes. Cette démarche consiste à observer certains « indicateurs » (travail, mariage, sexualité, virginité, contraception, rapport au religieux, maîtrise du français ...) en considérant que ceux-ci ne «s'améliorent » qu'en immigration ou parmi les descendantes de migrant'e's, par rapport aux valeurs transmises par leurs parents. L'usage de la contraception et la baisse de la fécondité sont dans ce cadre considérés comme de nouveaux comportements liés à l'immigration (Honoré-Castellin, 1990), bien que d'autres

\footnotetext{
${ }^{1}$ L'analyse de cette question prend place au sein d'une recherche de thèse dont l'objectif était d'étudier les effets empiriques de l'articulation des rapports sociaux de sexe, de "race » et de classe sur le regard porté sur les migrantes dans leur ensemble, et sur le travail et les attitudes de migrantes «seules » du Maroc sans-papiers en France (Moujoud, 2007). Le terrain investi pour cette recherche croise les deux sociétés, marocaine (Casablanca) et française (Paris et sa banlieue proche). Il se compose d'une centaine d'entretiens semi-directifs avec des migrantes « seules » sans-papiers ou régularisées, des migrantes rurales domestiques à Casablanca, des hommes migrants légaux en France et des représentant·e's d'associations de soutien aux migrant·e's.
} 
champs de recherche insistent sur la chute de la natalité à l'intérieur des sociétés d'émigration, comme Zahia Ouadah-Bedidi et Jacques Vallin (2000) pour le Maghreb ${ }^{2}$.

L'approche évolutionniste tend à produire l'idée qu'il serait nécessaire de rejeter les « valeurs » de la société d'origine afin de «s'intégrer » en France. Ce rejet serait l'attitude de descendantes de migrant ${ }^{\cdot} \cdot s$ qui auraient " évolué » par rapport aux réalités de leurs parents et de leur société d'origine, comme dans Yasmina et les autres de Nanterre et d'ailleurs, de Camille Lacoste-Dujardin : « Le plus élevé de tous les paramètres disposant à l'intégration est clairement la dépréciation, voire le rejet, des conditions de vie au Maghreb. » (1992 : 266267). L'idée de changement apparaît par opposition à « la société d'origine », associant ainsi tout ce qui est « traditionnel » (et oppressif) aux parents ou à cette société, et tout ce qui est « moderne » (et émancipateur) aux jeunes filles ou à la société d'immigration. Selon Sandrine Gaymard (2003), il s'agit pour les Françaises de parents maghrébins d'une: «situation conflictuelle entre d'une part la culture occidentale et la culture musulmane et d'autre part la première et la deuxième génération ». D'où par ailleurs l'idée de "perte de repères », ainsi que le formule Abdessalem Yahyaoui: «[Certaines migrantes] perdent les repères d'ici et de là-bas et $[\ldots]$ chutent sous la pression de désirs en contradiction et d'appels inquiétants : se laisser pénétrer par la modernité, changer ou rester les mêmes. » (1994 : 9). Il peut aussi être question d'une prise de conscience de la domination qui n'aurait lieu qu'en France ou sous l'influence du féminisme "occidental» (comme si les migrantes ne portaient pas ellesmêmes, aussi, le féminisme, et comme s'il n'existait pas de féminisme depuis longtemps dans le reste du monde). Ainsi, un lien "naturel» est établi entre changement (entendu comme développement) et vécu en France.

\section{B. La domination et les effets contradictoires de la migration}

D'autres travaux montrent qu'il est difficile de soutenir que la migration est en soi une émancipation de la domination. Les travaux sur l'histoire des migrantes en Europe insistent sur le fait que "l'équation émigration = émancipation» est "loin d'être automatique » (Morelli ; Gubin, 2004 : 12). Évoquant aussi bien le cas des épouses des immigrés qui restent au pays et «trouvent leur émancipation dans le départ de leur mari », que celui des migrantes qui partent seules et accèdent à l'autonomie financière. Anne Morelli et Eliane Gubin concluent que : «La migration féminine peut exacerber la dépendance de certaines mais être libératrice pour d'autres et elle peut finalement changer, peu ou prou, les rapports de genre au sein du couple et de la famille» (Morelli ; Gubin, 2004: 13). Du côté des travaux anglophones, les recherches féministes posent la question de la façon dont la migration internationale change les comportements de genre et permet «l'empowerment » des femmes. Selon Saskia Sassen (2005), leurs conclusions convergent: globalement, la migration contribuerait à l'autonomie des femmes, mais que les profits qu'elles en tirent resteraient limités.

Beaucoup de chercheuses indiquent que les migrantes sont soumises à la domination et utilisent des stratégies afin d'y échapper (Agustín, 2005 ; Morokvasič ; Catarino, 2005). Pour Morokvasič et Catarino, "les effets de la migration [en général] ne sont pas nécessairement empowering ou, tout du moins, ne se traduisent pas nécessairement par une modification des rapports sociaux de sexe au sein du couple ou de la famille ». D'après plusieurs auteur'e's

\footnotetext{
${ }^{2}$ L'une des explications de la prégnance de l'analyse évolutionniste ou en termes d'indicateurs prédéfinis distinguant les deux sociétés de départ et d'arrivée peut être la fragmentation entre les champs de recherche sur la migration internationale et sur telle ou telle société du « Sud ».
} 
travaillant sur le cas français, les rapports de genre se reconstruisent différemment en contexte migratoire grâce aux initiatives des migrantes. Sandra Laurain (2005) le démontre à partir de l'analyse de l'engagement militant des femmes mahoraises (comoriennes) en France. De même, pour Muriel Azoulay et Catherine Quiminal (2002), c'est sur la base de l'implication dans des associations que des migrantes africaines «peuvent entamer des négociations dans un rapport de forces qui leur soit moins défavorable que dans le seul face à face avec leur époux ». Les femmes deviendraient " "réveillées" 》 et les "hommes menacés » en milieu soninké en France. Dans le contexte migratoire, hommes et femmes recourent à des stratégies; les uns pour asseoir leur domination, les autres pour y échapper dans un cadre de tensions et de «rencontre entre deux modes de contrôle sur les femmes» (Azoulay; Quiminal, 2002:88).

Toutefois, la question des transformations des rapports de sexe est très souvent analysée par rapport à la « communauté » (nationale ou " ethnique ») migrante et/ou à l'intérieur du cadre familial, ce qui ne permet pas de considérer les rapports sociaux de sexe en général, ni les migrantes vivant ou/et ayant migré seules, hors famille. Cependant, quelques rares travaux sur les effets sexistes des lois migratoires (Lesselier, 2003), ainsi que ceux portant sur des migrantes sociologiquement minoritaires, comme les lesbiennes (Al'Rassace ; Falquet, 2007), les prostituées (Yun, Lévy ; Poisson 2006, Moujoud ; Texeira, 2005) ou les étudiantes (Bouly de Lesdain, 1999), commencent à enrichir l'analyse pour le cas français, en attirant l'attention sur des migrantes ne s'inscrivant pas dans le cadre familial. Ainsi par exemple, au lieu d'une amélioration de leur situation, les migrantes chinoises, irrégulières, souvent divorcées, qui se prostituent à Paris ou ont pour «débouché principal » le travail de services aux personnes chez des Chinois'es anciennement installé'e's en France, connaissent une déqualification par rapport à leur formation dans leur pays (Yun; Lévy ; Poisson, 2006). De même pour les jeunes camerounaises qui viennent en France avec un projet d'études et de promotion, mais se heurtent aux obstacles juridiques et économiques. Bouly de Lesdain (1999) explique qu'elles se retrouvent "enfermées » dans des emplois précaires qui ne correspondent pas à leurs aspirations d'avant la migration. Elles connaissent un processus de déclassement, à l'instar des migrantes haïtiennes que Rose-Myrlie Joseph (2007) a interrogées en France.

Ainsi, globalement, la question des liens entre les transformations que connaîtraient à la fois les migrantes et les femmes autochtones, celle des effets de la migration sur les rapports sociaux de sexe en général (et pas seulement dans la famille ou dans la "communauté » migrante), tout comme celle des formes que prennent ces transformations, sont rarement développées. Cela influe sur le regard global porté sur les effets de la migration sur les femmes, et conduit à (ou relève de) ce que le changement ne soit pas perçu sans immigration, c'est-à-dire dans les sociétés d'origine, qui demeurent présentées de manière figée.

\section{Persistance d'un regard réducteur sur les sociétés d'émigration}

Souvent, dans les travaux sur genre et migration, la société d'origine « se constate sans plus », ainsi que l'a déjà remarqué Sayad (1981) à propos de la recherche sur l'immigration. Les analyses concernent fréquemment des espaces définis de manière très imprécise (sociétés d'origine, sociétés du «Sud», les «autres sociétés») ou exagérément étendue (sociétés «africaines », «maghrébines ») ou encore désignées par des catégories réductrices ( "musulmanes », " arabes »). La démarche est étonnante : il est rare, par exemple, que les travaux sur la société française aillent chercher des références en Allemagne ou en Italie, malgré l'identification «occidentale » commune (et la présence importante d'une même 
religion chrétienne). De plus, cette démarche est simplificatrice. Elle procède de l'homogénéisation des " autres » sociétés, et par conséquent empêche de prendre en compte leur diversité (religieuse, linguistique...) et la pluralité des parcours et appartenances parmi les migrantes originaires d'un même pays.

Cette homogénéisation des sociétés d'origine ne permet pas non plus de considérer la diversité des positions sociales au sein du groupe femmes vivant dans une même société d'émigration. Cela se manifeste dans l'attention portée aux acquis des migrantes résultant de la migration, par opposition aux acquis des femmes dans " la société d'origine », et non par rapport à leur propre position sociale prémigratoire. En effet, beaucoup de travaux (portant ou non sur le cas français) concluent que les acquis des migrantes dépendent des rapports de genre dans leur société d'origine, ce qui risque de faire croire que le sexisme est localisé dans les sociétés d'émigration, alors que l'égalité des sexes serait intrinsèque aux sociétés d'immigration. Par exemple, les nouvelles recherches féministes (anglophones) s'accordent pour dire que les bénéfices de la migration pour les femmes varient selon leur société d'origine et le statut dans lequel elle les maintient (Patricia R. Pessar) ${ }^{3}$. Cette idée ignore l'hétérogénéité de chaque société d'origine et tend à rendre celle-ci seule responsable de la « non-émancipation » des migrantes.

Simultanément, en France, les "sociétés d'origine » sont largement associées aux pays du Maghreb, comme l'indiquent Stéphanie Condon (2000) et Catherine Raissiguier (2003). Bien qu'il existe de plus en plus des textes portant sur des migrantes de diverses régions du monde, les lectures majoritaires sur les migrantes ou leurs descendantes sont loin de prendre en compte toutes les femmes qui s'identifient ou peuvent être identifiées en tant que migrantes ou descendantes de migrant' $\cdot$ 's, notamment les Asiatiques, les Européennes ou les Latinoaméricaines. Par exemple, les travaux sur les Chinoises ou descendant'e's de Chinois'es sont très rares en France, alors que la migration chinoise dans ce pays n'est pas nouvelle. Elle représente même l'une des plus importantes concentrations de migrant' $\mathrm{e} \cdot \mathrm{s}$ chinois'e's en Europe (Yun ; Lévy ; Poisson, 2006 : 53).

En fait, la focalisation sur telle ou telle population migrante évolue selon les périodes historiques. L'attention académique portée aux « Maghrébines » date des débuts des années 1980, alors que les Portugaises étaient pourtant aussi nombreuses que les Algériennes ${ }^{4}$. Elle accompagne l'intérêt porté aux «Maghrébins » (progressivement considérés comme «les immigrés » par excellence) et s'accentue avec la préoccupation croissante des pouvoirs publics pour "l'insertion des jeunes générations". On remarque d'ailleurs une sorte de confusion entre «femmes immigrées » (ou descendantes de migrant'e·s) et Maghrébines (ou descendantes de Maghrébin'e·s) ${ }^{5}$ au point qu'Annette Goldberg-Salinas croit qu' « un grand problème que soulève l'analyse de la production académique et non académique dans ce domaine est celui de la généralisation abusive à toutes les "femmes immigrées" ou à toutes les "jeunes immigrées" [...] d'analyses et interprétations qui se réfèrent aux femmes et aux filles du monde arabo-musulman » (1996:36).

Selon Goldberg-Salinas, les analyses concernant les femmes du «monde arabo-musulman » sont étendues aux autres migrantes. Certes, la littérature la plus abondante sur le cas français

\footnotetext{
${ }^{3}$ Patricia R. Pessar constate que les « gains sont bien sûr variables selon les pays d'origine et selon le statut qu'ils réservent aux femmes » citée par Attias-Donfut ; Delacroix (2004: 183).

${ }^{4}$ Je remercie Stéphanie Condon qui me l'a rappelé.

${ }^{5}$ Cette confusion entre « immigrées » et «maghrébines » rappelle ce que décrit Nouria Ouali $(2002: 75)$ à propos du glissement de la signification du terme immigré en Belgique, qui devient synonyme de « musulman ».
} 
porte sur les Maghrébines ou les «musulmanes» «regroupées par leur mari » ou descendantes de migrant $\cdot e \cdot s$, au détriment des autres migrantes ou descendantes de migrant'e's. Toutefois, on pourrait aller plus loin que Goldberg-Salinas et se demander ce qui est désigné par «monde arabo-musulman» et si les catégories sur ce dit monde sont pertinentes. On peut penser qu'une partie non-négligeable reproduit les schémas de pensée issus du discours orientaliste et post-colonial, comme le démontre Meriem Rodary (2007) à propos de l'invisibilisation du travail des femmes populaires dans les sociétés colonisées, notamment marocaine.

\section{Les effets de la vision binaire qui sépare les sociétés de départ et d'arrivée}

La littérature sur «genre et migration » est souvent implicitement ou explicitement orientée par l'idée d'une opposition sommaire entre les sociétés de départ et d'arrivée. Cette vision binaire considère chacune des deux sociétés en fonction d'attributs tenus pour naturellement différents. C'est là un phénomène étonnant compte tenu de l'histoire commune, notamment coloniale pour les sociétés qui ont subi ou pratiqué la colonisation, de la mondialisation, des politiques de développement impliquant le genre, des luttes de femmes et des féministes et de la recherche scientifique développée dans les diverses sociétés d'émigration. La vision binaire dans les travaux sur "genre et migration » tend à opposer deux univers, occidental et non occidental, que tout séparerait, ce qui soulève plusieurs problèmes.

\section{A. L’occultation des dynamiques de genre dans les sociétés de départ}

En procédant d'une distinction sommaire entre les sociétés, la réflexion autour de la question des effets de la migration sur les migrantes ne considère pas les mutations dans les sociétés d'émigration, limitant le changement aux sociétés d'arrivée. Elle néglige souvent le fait que les sociétés du départ n'échappent pas aux transformations sociales que connaissent d'autres sociétés, oubliant que le départ même des migrantes s'effectue dans des sociétés en pleine transformation. Autrement dit, si la migration fabrique peut-être le changement, elle est aussi fabriquée par lui. Véronique Manry et Natalia Ribas-Mateos (2005) rejoignent cette critique lorsqu'elles invitent les spécialistes de la migration à analyser la mobilité des femmes comme « baromètre des transformations sociales et culturelles des sociétés d'origine ». N'empêche, de nombreux travaux continuent à ignorer le changement et les luttes de femmes, ethniques et de classes dans les sociétés de départ.

Dans le même registre, mais dans une étrange inversion des réalités empiriques, la non-prise en compte des transformations que connaissent les sociétés d'émigration conduit parfois à penser que les changements dans les sociétés d'origine seraient dûs... à la migration ellemême. Nous pouvons le lire chez Angeles Ramírez (1999), pour qui les changements qu'apporte la migration dans la vie des Marocaines en Espagne vont influencer le Maroc, où selon elle le travail des femmes est mal vu notamment du fait de l'islam - le prisme religieux se substituant ici à l'analyse en termes de division sexuelle de travail ${ }^{6}$. Si la question des effets de la migration sur le « changement positif» dans les sociétés d'origine est importante à soulever, elle demeure difficile à aborder en l'absence de travaux spécialisés centrés sur ces sociétés, et non pas seulement sur les sociétés d'immigration. Je me contente ici d'attirer l'attention sur la vision réductrice qui souligne l'éventuelle influence des migrantes

\footnotetext{
${ }^{6}$ On retrouve ici une continuation des préjugés sur le non-travail des Marocaines et des «Orientales », que Meriem Rodary (2007) et Fatema Mernissi ont déconstruits dans plusieurs de leurs travaux.
} 
« émancipées » sur leur société d'origine, sans considérer le changement propre à cette société, ainsi que les divers facteurs (luttes, politiques nationales et internationales, etc.) qui agissent sur place.

On retrouve souvent, dans les travaux récents sur le transfert international du travail de reproduction, cette occultation du changement dans les sociétés d'origine. Ces travaux tendent à expliquer la migration des « femmes du Sud» uniquement par la position des «femmes du Nord ${ }^{7}$. Celles-ci se libéreraient de certaines tâches domestiques afin de «mener une vie active et sociale hors du foyer », en déléguant ces tâches aux "femmes du Sud » (Manry et Ribas-Mateos, 2005) ${ }^{8}$. Pourtant, si l'on prend en compte les résultats des nombreux travaux sur les domestiques ou sur le travail des femmes «qualifiées » au « Sud », on constate que des femmes riches «se libèrent» du travail domestique aussi bien au «Sud» qu'au "Nord» (Moujoud, 2007).

\section{B. La construction d'une opposition « nous »/« elles »}

Dans l'ensemble, le langage fondé sur la binarité entre deux sociétés de départ et d'arrivée renvoie d'emblée les (é)migrantes à une altérisation radicale. Parfois, ce langage est en plus évolutionniste, comme on l'a déjà vu. Il postule que pour s'émanciper, les migrantes doivent s'affranchir des éléments culturels de leur société d'origine et intégrer ceux des femmes de la société d'arrivée. Les (é)migrantes sont dans le même mouvement présentées comme singulièrement aliénées : soumises ${ }^{9}$, cloîtrées, ne travaillant guère... D'autres fois, dans le sillage des travaux critiques de l'évolutionnisme, apparaît la tendance au relativisme culturel, qui distingue les «femmes du soi » des «femmes de l'autre», comme dirait Nicole-Claude Mathieu (1987). Comme la vision évolutionniste, il procède d'une logique binaire d'opposition entre femmes et entre sociétés. Ce relativisme apparaît dans l'idée de respecter la différence des « autres », ou encore de les laisser inventer leur "propre » féminisme (comme si le féminisme n'avait pas déjà une longue histoire "chez elles » et n'était pas traversé, comme partout ailleurs, d'oppositions liées à la classe, à la « race », à la sexualité, à l'attitude par rapport à la religion, etc.), par exemple - idée que l'on retrouve dans des analyses sur les migrantes, les descendantes de migrants ou les femmes « non occidentales ».

Dans ces deux approches, les situations des (é)migrantes sont perçues comme «une » expérience naturellement différente de celles des "Occidentales». On constate ici les problèmes de simplification, de généralisation et de stéréotypes (donc du racisme comme le définit Colette Guillaumin, 1983). À ces problèmes, s'ajoute l'absence de comparaisons rigoureuses entre la position des (é)migrantes et celle des non-migrantes. La littérature sur genre et migration ne développe pas d'analyses sur les liens ou les différences entre les changements qui affectent les unes et les autres. On constate cette absence de perspective comparative ailleurs dans les schémas déjà critiqués par Laura Nader (2006), schémas comportant des dichotomies qui empêchent de " comparer véritablement » les positions des «Occidentales» et des « Orientales ». En se contentant d'opposer (et d'homogénéiser) les sociétés de départ et d'arrivée, la lecture fondée sur la vision binaire éloigne non seulement la

\footnotetext{
${ }^{7}$ Le rôle des Etats et des hommes du «Nord» dans le travail domestique est largement occulté par ce type d'analyse.

${ }^{8}$ Ces dernières expressions sont tirées du texte de Manry et Ribas-Mateos (2005).

9 Jean Guyot, Ruth Padrun, Évelyne Dauphinet et Yvone Jospa (1977: 140-149) évoquent des images de femmes enfermées dans la société d'origine " pour qui [...] la liberté ne veut rien dire ». Pour Gaymard (2003 : 30), «Les femmes [musulmanes] sont habituées dès leur enfance à être inférieures aux hommes. D’un point de vue personnel, une jeune fille musulmane souffre moins d'une situation qui serait intolérable aux yeux d'une jeune européenne actuelle. »
} 
possibilité de comparer, mais aussi d'interroger comment les migrantes défavorisées de diverses origines géographiques partagent entre elles et avec des femmes autochtones des conditions concrètes d'exploitation et de domination, et éventuellement des formes de luttes. Aussi pose-t-elle la question de l'occultation de la domination.

\section{L'occultation de la pluralité, de la continuité et de l'imbrication des rapports sociaux de pouvoir}

La lecture binaire peine à penser que les migrantes se heurtent à la production légale de l'oppression dans le contexte de la mondialisation néolibérale associant les dominations de classe, de sexe et de " race » (qui inclut aussi, la nationalité et le statut légal). Les effets de ces trois rapports sociaux de pouvoir, qui s'articulent (Falquet, 2006), se caractérisent pas la continuité ; ils se retrouvent tant dans la société de départ que dans celle d'arrivée. Comme le démontre Paola Tabet, dont les travaux partent d'une réflexion sur l'échange économicosexuel et la mobilité géographique des femmes (2004), les migrations féminines se situent à l'intersection d'un " enchevêtrement complexe de facteurs » (Tabel, 2004 : 139), construits par les rapports sociaux de sexe, les politiques et l'économie locales et internationales, ainsi que par les motivations et les objectifs individuels. Tabet indique ainsi que pour les femmes, le fait de partir, celui de choisir (de se prostituer ou pas ou encore de résister) s'inscrivent dans cet enchevêtrement de facteurs : "C'est là qu'ils [ces faits] deviennent possibles ou qu'ils sont carrément créés, c'est là qu'ils sont stimulés au moyen d'offres de travail et de solutions meilleures, mais c'est là aussi que des formes de travail forcé sont imposées aux femmes recrutées par la contrainte ou l'abus de confiance.»(2004: 139). Ainsi, Tabet s'éloigne de certaines analyses féministes qui situeraient la lutte et le départ des migrantes dans la domination des femmes uniquement dans les sociétés d'origine. Elle pose comme « remarque préalable », le « rapport entre le local et le global » (2004 : 135).

Ce rapport n'apparaît pas dans les travaux qui analysent le changement dans la vie des migrantes à travers l'opposition société de départ/société d'arrivée, ou seulement à travers le changement dans la famille ou le groupe d'origine. Ces travaux laissent dans l'ombre le rapport historique (notamment colonial-capitaliste) et les formes de «modernité » (industrialisation, salariat, urbanisation, exploitation, élites post-coloniales, etc.) que ce rapport a façonné dans les sociétés colonisées devenues sociétés d'émigration. Ils mettent de côté le fait que 1) bien que des migrantes soient originaires d'un même pays, elles ne sont pas de la même classe sociale que d'autres femmes originaires ou vivant dans ce pays ; 2) la domination ne naît pas que du couple ou du groupe d'origine : elle apparaît dans les lois migratoires racistes qui renvoient les migrant'e's aux cadres normatifs conjugaux et familiaux, en leur refusant l'autonomie juridique (Lesselier, 2003) ; 3) les États d'origine et d'arrivée oppriment conjointement les migrantes, ce qui se manifeste notamment dans l'existence d'accords bilatéraux sur le statut personnel (Haoua Lamine, 2002), par exemple, et à travers le contrôle par les divers pays de la mobilité des défavorisé'e·s.

Donc, lorsqu'elle se fixe d'emblée sur les acquis des migrantes dans la société d'arrivée, en opposition à la société de départ, et au sein du groupe migrant, la lecture des effets de la migration sur les migrantes ne peut saisir comment le contexte (du départ et d'arrivée) permet à ces femmes de s'émanciper ou non. Il est ainsi pour le moins problématique de célébrer comme un acquis l'accès au travail salarié en migration, tout en négligeant les caractéristiques du marché du travail dont il s'agit et les déqualifications que peuvent connaître les migrantes par rapport à leur société d'origine. Cela mène à oublier que les migrantes (défavorisées du 
Sud) ne parviennent à une certaine autonomie que dans les limites où leurs luttes leur permettent de manipuler les contraintes sur les deux fronts de leur parcours migratoire, d'abord pour partir (donc pour fuir) et puis pour lutter (donc pour rester et peut-être s'émanciper). Et c'est pourquoi la migration prend chez elles la forme de pénibles épreuves, notamment pour celles que les politiques des États ont placées en situation irrégulière, les amenant à consacrer l'essentiel de leurs efforts quotidiens à rechercher des ressources dans un contexte de "non-droit aux droits», pour reprendre la formule de Marie-Claire CalozTschopp (2006) sur les situations des clandestines en Europe.

\section{La migration peut-elle être un facteur d'autonomisation?}

La migration peut-elle être un facteur d'autonomisation des femmes dès lors qu'elle s'inscrit dans des cadres oppressifs et que les limitations sont nombreuses dès que les femmes dominées du fait de leur sexe, leur classe et leur « race » veulent traverser les frontières entre États ? Que révèle la migration des femmes parties seules sans papiers sur cette problématique?

Ces questions dépassent bien sûr les limites de ce texte. Je propose toutefois de passer de l'approche binaire à une analyse en termes de dialectique entre domination et résistance dans le cadre de la mondialisation néolibérale. Cette perspective permettra non seulement de saisir les effets empiriques de l'articulation des rapports sociaux de sexe, de classe et de "race ", mais de ne pas victimiser les migrantes et de considérer leurs luttes. La perspective internationale permet d'aller au-delà de la vision binaire. Elle conduit à tenir compte du fait que les éléments que l'on peut prendre pour indices d'émancipation par la migration 1) font probablement partie des réalités dans les sociétés d'origine; 2) sont le fruit de luttes individuelles et collectives, et non pas de quelconques facilités qui seraient offertes d'emblée par la société d'immigration aux (é)migrantes.

\section{A. Entre le contrôle du groupe et celui des États}

Dans l'ensemble, sans préjuger d'autres motifs de migration, les migrantes «fuient» le sexisme de leur société d'origine, comme l'a démontré Mirjana Morokvasic (1986). Toutefois, le cas des migrantes «seules" sans-papiers montre que le contrôle ne naît pas que du groupe d'origine (et même que celui-ci peut soutenir le projet migratoire), et que, du coup, pour les femmes dominées du fait de leur classe, leur sexe et leur " race », la quête de l'autonomie doit prendre en compte plusieurs facteurs.

En effet, le projet de départ des migrantes seules du Maroc vers la France résulte d'une quête d'autonomie commencée dans la société d'origine où ces femmes envisagent le départ comme une alternative aux positions subalternes qu'elles subissent (Moujoud, 2007). Cette situation concerne d'autres migrantes internationales, comme le montrent Chantal Saint-Blancat (1997) sur les Marocaines en Vénétie, ou encore Liane Mozère (2005) sur le projet migratoire des Philippines domestiques à Paris. Pour Mozère, il s'agit d'un « véritable projet de vie dont toutes les femmes rencontrées font état et qui structure, soutient et légitime le processus migratoire $»$.

Dans la situation de migration seule, situation que l'on pourrait penser hors de la famille, le projet migratoire est non seulement individuel mais aussi collectif et très souvent familial (Moujoud, 2007). D'abord, les émigrantes l'ont souvent initialement fait porter par leurs proches, qui les ont matériellement soutenues pour partir en dépit des restrictions étatiques. 
Puis, les migrantes accordent de l'importance aux solidarités matérielles qu'elles construisent à l'égard de leurs parents, enfants, amies, etc. D'un côté, cela montre que si, sous l'effet notamment de la distance géographique, les migrantes s'émancipent du contrôle par l'institution familiale de naissance, cette émancipation ne naît pas de l'immigration; le départ des femmes seules étant lui-même révélateur de la réduction du contrôle de leur mobilité par la famille (qui soutient souvent le départ). Mais de l'autre côté, cela implique que le contrôle des femmes prend de nouvelles formes lorsqu'elles décident de partir. Il se double d'une dimension de contrôle non pas familial, mais étatique, conduisant les femmes défavorisées à lutter pour avoir le droit à la mobilité internationale, puis pour vivre et travailler légalement dans la société d'immigration.

Tout au long de leur parcours migratoire, les migrantes « seules » sans papiers se situent entre restrictions légales et quête d'autonomie. Elles acquièrent une certaine autonomie (en partant et en vivant seules, par exemple), construisent un projet de vie marqué par la distanciation (mais pas la rupture) par rapport au milieu d'origine, mais n'échappent guère à la domination et aux normes sexistes. L'irrégularité administrative se conjugue souvent dans leur quotidien à d'autres statuts dévalorisants, comme la prostitution, la domesticité, le non-mariage, le veuvage, le divorce ou le non-emploi. Ces statuts renforcent la stigmatisation qu'elles affrontent en tant que femmes seules (Pheterson, 2001), alors même que la peur (de la police notamment) détermine leurs attitudes, comme elle marque la vie des autres femmes sanspapiers en France (Domingues; Lesselier, 2006). En même temps, la mise en couple (en particulier hétérosexuel et par le mariage) devient le principal recours pour accéder aux droits au séjour et donc au travail protégé, à la santé, aux formations, à la mobilité géographique, etc. Pour éviter la stigmatisation et obtenir des droits sociaux et politiques en France, les femmes ont intérêt à se marier, de préférence avec un homme privilégié du fait de sa " race ", son statut légal et sa nationalité. Aussi, en situation de migration seule et d'irrégularité administrative, l'institution du mariage est imposée aux femmes par les deux sociétés de départ et d'arrivée et devient l'objet d'un double contrôle. Bien que les migrantes parties seules se dégagent plus ou moins du contrôle familial, elles n'en sont pas moins soumises au contrôle des lois du groupe d'origine (société, État, consulat, etc.) autant qu'à celui de la société française (lois migratoires, contrôle policier, nouvelle famille, etc.).

Accéder à un statut légal devient la principale voie d'autonomisation en immigration pour les migrantes « seules », pauvres et racisées, et donc illégalisées - tout au moins pour une période de leur parcours migratoire. À partir du moment où elles sont régularisées, elles jouissent enfin de droits sociaux et politiques nécessaires à leur autonomisation. Elles obtiennent donc une autonomie qui s'établit à partir des logiques instituées par les lois migratoires, et non pas « culturelles », "sociales» ou religieuses. Les migrantes régularisées sont (et se sentent) libérées du contrôle policier, moins vulnérables par rapport à l'exploitation dans le travail salarié et valorisées par leur milieu d'origine du fait de leur nouveau statut légal et des ressources qu'il leur accorde. Car, dans le cadre des migrations soumises à l'illégalisation, n'a échoué dans son projet migratoire (et son autonomie) que le/la migrant·e qui ne réussit pas à régulariser son statut légal. Les migrantes régularisées sont mieux armées pour nourrir les solidarités matérielles avec les proches et montrer la réussite du projet migratoire - les sanspapiers étant astreint $\cdot e \cdot s$ à l'immobilité. Nous arrivons par là à l'explication de la manière par laquelle la « race » ou le statut légal complètent ou se substituent à la domination masculine (notamment du groupe d'origine) tout en se fondant sur les mêmes logiques de contrôle et d'oppression. Les lois migratoires racistes empêchent l'autonomisation des migrantes par la migration, comme elles répriment leurs aspirations et leur barrent la voie de la reconnaissance dans les deux sociétés de départ et d'arrivée. 


\section{B. Se « débrouiller » individuellement}

Bien que les migrantes s'organisent éventuellement avec le soutien d'autres acteurs-rices, politiques et sociaux-elles, dans le contexte d'illégalisation, les limitations de droits génèrent fréquemment des attitudes de débrouille individuelle. L'illégalisation ne laisse aux (é)migrantes que peu d'issues, les forçant à s'orienter vers le travail non protégé, la prostitution ou le mariage plus ou moins «blanc », afin de voyager et/ou de rester et vivre, surtout légalement, en immigration. Certes, les migrantes en situation irrégulière recherchent autonomie matérielle et possibilités d'être soustraites à la pression sociale de leur groupe d'origine, mais de manière informelle, indépendamment des institutions de la société d'immigration et souvent même contre elles. Elles s'appuient sur leurs relations sociales amicales, amoureuses, professionnelles et familiales. Autrement dit, les voies vers l'autonomie ne relèvent pas de structures qui seraient fournies par l'État et proposées aux (é)migrantes (pour migrer, travailler ou s'émanciper). Ces femmes inventent des ressources en s'adaptant aux secteurs qui leur sont laissés. Elles peuvent cumuler des stratégies plurielles, dont on peut schématiser les dimensions essentielles autour de quatre aspects principaux :

- économique: les migrantes irrégulières sont conduites à rechercher leur survie matérielle et financière dans des secteurs sexués, ethnicisés, informels et non protégés (prostitution, domesticité non-déclarée, travail dans des « ateliers clandestins », des entreprises familiales, commerce informel, etc.).

- juridique : elles sont amenées à focaliser leur attention sur les possibilités de régulariser leur statut légal et d'accéder aux droits en tant que régulières ou nationales.

- social : elles sont amenées à contrer les rapports sociaux en s'appuyant seulement sur les relations sociales. Elles reconvertissent les liens sociaux (amicaux, amoureux, sexuels ou de travail, par exemple) en liens de solidarité dans les divers domaines de leur vie (logement, santé, travail, régularisation, contact avec les proches, etc.).

- associatif et militant: les migrantes sans-papiers se mobilisent dans le cadre des actions en faveur des migrantes, actions qui leurs fournissent un espace nécessaire de soutien et d'informations ${ }^{10}$.

Plusieurs pistes restent à creuser. Les migrantes ont des trajectoires et des statuts variés. Les recours qu'elles empruntent sont donc probablement plus divers dans leurs modalités, loin d'être spécifiques à la population migrante et beaucoup plus contrastés que ne le laisse supposer toute description figée. La réflexion sur les effets de la migration sur les femmes gagnera peut-être à se détourner des perceptions simplistes des rapports de genre (limités au contrôle par le groupe d'origine ou familial) et de l'autonomie (fondée sur des oppositions entre sociétés ou sur des « indicateurs » prédéfinis, comme «l'accès au travail »). S'en tenir aux rapports de genre au sein de la famille ou de la «communauté » migrante ne permet pas de rendre compte de la diversité des migrantes et de leur relation aux structures familiales, ni de comprendre l'inscription de la migration des femmes dans la continuité historique de la colonisation et dans la mondialisation néolibérale. Cette démarche limite la domination des femmes, ainsi que leurs marges de manœuvre aux frontières «ethniques » ou familiales, et non pas à d'autres modalités de la domination, notamment raciste et étatique, tout en invisibilisant les effets du sexisme des sociétés d'arrivée. Mais ce constat ne saurait donner

\footnotetext{
${ }^{10}$ Le contexte politique répressif restreint l'engagement associatif des migrantes sans papiers que j'ai rencontrées. Celles-ci peuvent craindre une visibilisation de leur présence irrégulière en France ou ont eu l'occasion de saisir les limites imposées à ces actions et la nécessité d'inventer d'autres luttes (Moujoud, 2007).
} 
quitus à certains travaux qui, en insistant sur la domination, ne laissent paraître ni les résistances des migrantes et des racisées, ni leurs apports aux luttes des femmes autochtones et en direction de leur pays d'origine, comme on peut le constater dans les travaux de Nadia Châabanne (2004) et dans les apports théoriques et politiques du Groupe du 6 novembre (2001).

Les nouvelles recherches pourront peut-être se pencher davantage sur les effets contradictoires de la migration: les femmes souhaitent renforcer leur autonomie et leur chance de mobilité sociale, mais sont en même temps enfermées dans des structures de domination dont elles doivent manipuler les règles. On pourrait s'intéresser à la manière dont déclassement et capacité d'agir coexistent chez des migrantes vivant en situation de promotion ou de déqualification par rapport à leur projet initial et à leurs qualifications dans leur société d'origine. On pourrait également situer ces effets contradictoires par rapport à la situation des femmes autochtones et adopter une perspective comparative, en analysant les changements liés à la migration dans un cadre plus général, non limité aux structures du groupe d'origine mais relié à la théorie des rapports sociaux de pouvoir et de leurs dynamiques. Cette théorie telle que définie par Danièle Kergoat (2000 et à paraître), nous fournit de quoi comprendre l'autonomisation des migrantes sans oublier leur position dominée ou idéaliser leur empowerment par la migration. L'analyse de l'autonomisation des migrantes pourra ainsi se référer aux solidarités collectives, aux luttes qui peuvent réunir migrantes et autochtones et aux relations interpersonnelles qu'elles construisent —et non pas à des indicateurs tous faits, définis en fonction de présupposés sur le groupe ou la société d'origine, ou à des «améliorations » qui seraient accordées par la société d'immigration ou nécessairement opposées à la vie dans la société d'émigration.

Aller au-delà de la vision binaire et des analyses centrées seulement sur la famille ou le groupe dit d'appartenance est donc une condition nécessaire à la lecture des effets de la migration sur les rapports sociaux de sexe. La perspective internationale et comparative est susceptible de le permettre. Elle conduira à ne pas centrer le regard sur la seule immigration, à mieux réfléchir sur les transformations qui sous-tendent la migration des femmes, à prendre en compte les similarités des dynamiques de genre dans les sociétés de départ et d'arrivée, et à saisir par conséquent ce que le changement dans la vie des migrantes doit ou non à la migration internationale. Cela nécessite de faire retour sur les transformations politiques et socioéconomiques dans les sociétés d'émigration, et donc sur les implications genrées de la colonisation, de la mondialisation et des "politiques de développement », ainsi que sur les luttes de femmes dans ces pays, leurs acquis et les contradictions en leur sein, et non seulement au sein du groupe femmes dans les sociétés d'arrivée. Car, ce qui pose problème dans la vision binaire, c'est d'abord le point de départ basé sur des dichotomies renvoyant à deux sociétés l'une et l'autre figée. J'ai tenté de dire qu'il est nécessaire de rompre avec cette perspective théorique et de réfléchir sur de nouveaux paradigmes afin de reformuler la problématique des effets de la migration sur les femmes, en regardant du côté de la dialectique entre domination et résistance dans le cadre de la mondialisation néolibérale.

\section{Bibliographie}


AGUSTIN, Laura Maria (2005), « Cessons de parler de victimes, reconnaissons aux migrants leur capacité d'agir », Cahiers genre et développement, n 5, p. 109-115.

Al RASSACE, Sabreen et FALQUET Jules (2007), « De la nécessité de faire bon accueil aux lesbiennes et aux femmes étrangères. Lesbiennes étrangères en mouvement en France aujourd'hui », in: Freedman, jane ; Valluy Jérôme (coords.). Persécutions des femmes. Savoirs, protections et mobilisations, Paris : Editions du Croquant, p. 339-372.

AL-SADDA, Hoda (2004), «Le discours arabe sur l'émancipation féminine au $20^{\mathrm{e}}$ siècle », Vingtième Siècle, Revue d'histoire, $\mathrm{n}^{\circ}$ 82, p. 81-89.

ATTIAS-DONFUT, Claudine ; DELACROIX, Catherine (2004), « Femmes immigrées face à la retraite », Retraite et Société, ${ }^{\circ}$ 43, p. 138-163.

AZOULAY, Muriel ; QUIMINAL, Catherine (2002), « Reconstruction des rapports de genre en situation migratoire. Femmes "réveillées", hommes menacés en milieu soninké ", Vei enjeux, $\mathrm{n}^{\circ} 128$, p. 87-101.

BOULY de LESDAIN, Sophie (1999), « Projet migratoire des étudiantes camerounaises et attitude face à l'emploi », Revue Européenne des Migrations Internationales, (15), 2, p. 189202.

CALOZ-TSCHOPP, Marie-Claire (2006), «Clandestinité des femmes migrantes. Apartheid de sexe, violence, globalisation », Vivre Clandestine, Collectif Femmes en noir contre les centres fermés et les expulsions, Université des femmes, Bruxelles, p. 63-113.

CATARINO, Christine ; MOROKVASIČ, Mirjana (2005), «Femmes, genre, migration et mobilité », Revue Européenne des Migrations Internationales, (21), n 1, p. 7-27.

CHÂABANE, Nadia (2004), « Là-bas et ici », sur http://www.mediterraneas.org (consulté le 20 octobre 2005).

CONDON, Stéphanie (2000), «L'activité des femmes immigrées du Portugal à l'arrivée en France, reflet d'une diversité de stratégies familiales et individuelles », Population (55), 2, p. 301-330.

DOMINGUES, Clara ; LESSELIER, Claudie (2006), « Sans-papiers et institutions françaises - paroles de sans-papières » sur http://www.femmes-histoire-immigration.org (consulté le 2012-2006).

FALQUET, Jules (2006), «Hommes en armes et femmes "de service" : tendances néolibérales dans l'évolution de la division sexuelle et internationale du travail », Cahiers du Genre, Travail et mondialisation. Confrontations Nord/Sud, n 40, p. 15-38.

GAYMARD, Sandrine (2003), La négociation interculturelle chez les filles francomaghrébines. Une étude de représentation sociale, Paris, L'Harmattan.

GROUPE du 6 NOVEMBRE (2001), Warriors/Guerrières, Paris, Nomades' Langues Editions.

GUILLAUMIN, Colette (1983), « Porter aux nues et penser pis que prendre. Remarques sur les stéréotypes dans le racisme », Recherche, Pédagogie et Culture, n 62, p. 35-39.

GUYOT, Jean; PADRUN, Ruth; DAUPHINET, Évelyne et al. (1977), Des femmes immigrées parlent, Paris, L'Harmattan-CETIM.

KERGOAT, Danièle (2000), "Division sexuelle du travail et rapports sociaux de sexe », in Helena Hirata, Françoise Laborie, Hélène Le Doaré, Danièle Senotier (dir.), Dictionnaire critique du féminisme, Paris, PUF, p. 35-34.

KERGOAT, Danièle (à paraître), « Dynamique et consubstantialité des rapports sociaux », in Dorlin, Elsa (coord.) « Sexe, classe, race. Pour une épistémologie de la domination », Paris, PUF.

LACOSTE-DUJARDIN, Camille (1992), Yasmina et les autres de Nanterre et d'ailleurs. Filles de parents maghrébins en France, Paris, La Découverte.

LAMINE, Haoua (2002), "Femme et étrangère, parfois la double discrimination », Réalités Familiales, $\mathrm{n}^{\circ} 64-65$, p. 62-69. 
LEMERCIER, Elise (2005), «La médiation sociale culturelle: du "sale boulot" à l'engagement collectif », Migrations société, (17), 99-100, p. 119-130.

LESSELIER, Claudie (2003), «Femmes migrantes en France : le genre et la loi » in Hersent Madeleine et Zaidman Claude (coord.), Genre, travail et migrations en Europe, Cahiers du CEDREF, p. 45-61.

MANRY, Véronique ; RIBAS-MATEOS, Natalia (2005), «Mobilités au féminin, ou comment appréhender la place des femmes et des mobilités dans la globalisation » Mobilités au féminin, Journées d'études, 15-19 novembre 2005, Tanger, sur http://www.mmsh.univaix.fr (consulté le 20 novembre 2005).

JOSEPH, Rose-Myrlie (2007), Genre, travail et migration : le cas des femmes haïtiennes en France. Mémoire de Mastère 1, Université Paris Diderot.

MATHIEU, Nicole-Claude (1987), «Femmes du soi, femmes de l'autre », Vers des sociétés pluriculturelles : études comparatives et situation en France. Actes du Colloque International de l'AFA, Paris, 9, 10, 11 janvier 1986, p. 604-614.

MERNISSI, Fatema (1987), "État, planification et discours scientifique sur la femme », in Mohamed Alhyane et al., Portraits de femmes, Casablanca, Le Fennec, p. 69-105.

MORELLI, Anne; GUBIN, Eliane (2004), "Pour une histoire européenne des femmes migrantes $»$, Sextant, $\mathrm{n}^{\circ}$ 21-22, p. 7-15.

MOROKVASIČ, Mirjana (1986), «Émigration des femmes: suivre, fuir ou lutter», Nouvelles Questions Féministes, n ${ }^{\circ}$ 13, p. 65-75.

MOUJOUD, Nasima (2007), Migrantes, seules et sans droits, au Maroc et en France. Dominations imbriquées et résistances individuelles. Thèse d'anthropologie sociale, EHESS.

MOUJOUD, Nasima; TEXEIRA, Maria (2005), " Migration et trafic de femmes » in Handman, Marie-Elisabeth; Mossuz-Lavau, Janine, La prostitution à Paris, Paris, La Martinière, p. 377-395.

NADER, Laura (2006), " Orientalisme, occidentalisme et contrôle des femmes », Nouvelles Questions Féministes (25), 1, p. 12-24.

OSO, Laura ; CATARINO, Christine (1997), «Les effets de la migration sur le statut des femmes. Le cas des Dominicaines et des Marocaines à Madrid et des cap-Verdiennes à Lisbonne » Migrations Société (9), $\mathrm{n}^{\circ}$ 52, p. 115-130.

OSO-CASAS, Laura (2007), «Femmes actrices des mouvements migratoires », sur http://www.iued.unig.ch (consulté le 15 mai 2007).

OUADAH-BEDIDI, Zahia ; VALLIN, Jacques (2000), « Maghreb : la chute irrésistible de la fécondité », Population et sociétés, $\mathrm{n}^{\circ} 359$, p. 1-4.

OUALI, Nouria (2002), "Politique migratoire et femmes migrantes en Belgique» in Mohamed Charef, Les Migrations au féminin, Agadir, Université Ibn Zohr, Les éditions Sud Contact, p. 61-83.

PHETERSON, Gail (2001), Le Prisme de la prostitution, Paris, L'Harmattan.

RAMÍREZ, Ángeles (1999), « La valeur du travail. L'insertion dans le marché du travail des immigrées marocaines en Espagne », Revue Européenne des Migrations internationales, (15), $\mathrm{n}^{\circ} 2$, p. 9-36.

RODARY, Meriem (2007), «Le travail des femmes dans le Maroc précolonial, entre oppression et résistance. Droit au travail ou accès aux bénéfices? », Cahiers d'études africaines, $\mathrm{n}^{\circ} 187-188$, p. 753-780.

SAINT-BLANCAT, Chantal (1998), «Les Marocaines en Vénétie : le changement sans ruptures », Migrations Société, (10), n 55, p. 107-115.

SASSEN, Saskia (2005), «Restructuration économique mondiale et femmes migrantes : nouveaux espaces stratégiques de transformation des rapports et identités de genre » (traduit de l'espagnol par Yves Le Scieller) in Genre, nouvelle division internationale du travail et migrations, Cahiers genre et développement, $\mathrm{n}^{\circ}$ 5, p. 103-108. 
SAYAD, Abdelmalek (1981), «Le phénomène migratoire: une relation de domination », Annuaire d'Afrique du Nord 1981, p. 365-406.

TABET, Paola (2004), La grande arnaque. Sexualité des femmes et échange économicosexuel, Paris, l'Harmattan.

YAHYAOUI, Abdessalem (dir.) (1994), Destins de femmes, réalités de l'exil. Interaction mère-enfants, Paris, La Pensée sauvage.

YUN, Gao ; LEVY, Florence ; POISSON, Véronique (2006), « De la migration au travail. L'exploitation extrême des Chinois-e-s à Paris », Travail, genre et sociétés, n 16, pp. 53-74. 